that GFAP might be released from these tumors, Jung et al. examined the levels of GFAP in the serum of patients with GBM and in two control groups, to determine the value of GFAP as a diagnostic marker of GBM.

This study included 98 consecutive patients with an initial diagnosis of solitary supratentorial intracerebral mass lesion who were admitted for surgery at the Medical Faculty of the Johann Wolfgang Goethe University, Frankfurt am Main, Germany. Neuropathological analyses revealed that 50 of these patients had a GBM, 31 had a non-GBM astroglial tumor, and 17 had a single cerebral metastasis of a primary noncerebral tumor. A further 50 healthy controls were included in the study.

The median serum GFAP level was significantly higher in patients with GBM $(0.18 \mu \mathrm{g} / \mathrm{l}$, range $0-5.6 \mu \mathrm{g} / \mathrm{l})$ than in non-GBM patients $(0 \mu \mathrm{g} / \mathrm{l}$, range 0-0.024 $\mu \mathrm{g} / \mathrm{l})$; GFAP was undetectable in all healthy controls. Both tumor volume and tumor necrosis volume were significantly correlated with serum GFAP level in patients with GBM $(P<0.001$ and $P=0.004$, respectively). A cutoff point for serum GFAP of $0.05 \mu \mathrm{g} / \mathrm{l}$ had a sensitivity of $76 \%$ and a specificity of $100 \%$ for the differentiation of GBM patients from non-GBM patients and healthy controls. The authors conclude that serum GFAP could be a useful new diagnostic biomarker for GBM.

Original article Jung CS et al. (2007) Serum GFAP is a diagnostic marker for glioblastoma multiforme. Brain 130: 3336-3341

\section{A new MRI protocol for detection of high-risk aortic plaques in patients with stroke}

Transesophageal echocardiography (TEE) is used to detect high-risk cardiac sources of cerebral embolism that could cause stroke, but it has limited diagnostic accuracy. Harloff et al. proposed and tested a new three-dimensional (3D) MRI protocol as an alternative to TEE for the detection of aortic pathologies in patients with acute stroke.

This study enrolled nonconsecutive patients with acute brain ischemia who underwent 3T $\mathrm{MRI}$, and cross-sectional and longitudinal TEE, of three aortic segments (ascending aorta, aortic arch and descending aorta) within a median of 5 and 6 days after hospitalization, respectively. Electrocardiogram-synchronized T1-weighted 3D MRI was performed on each aortic segment to identify plaques of $\geq 3 \mathrm{~mm}$ thickness; patients with such plaques underwent contrast-enhanced 3D magnetic resonance angiography. Breath-held T2-weighted and time-resolved images were also obtained at previously detected plaque locations.

TEE and MRI data were obtained from a total of 167 assessable aortic segments in 74 patients. The MRI protocol detected high-risk aortic plaques ( $\geq 4 \mathrm{~mm}$ ) in significantly more patients than did TEE (37 vs 23; $P=0.029$ ), as well as a greater absolute number of high-risk plaques and thrombi (74 vs 47). Routine diagnostics including TEE could not determine the etiology of stroke in 26 patients; among these 26 patients, MRI detected a high-risk aortic source of brain embolism in 8 (30.8\%), and 19 (73.1\%) experienced embolic brain ischemia. $\mathrm{MRI}$ overlooked high-risk plaques detected by TEE in only four patients.

The authors conclude that the proposed 3D $\mathrm{MRI}$ protocol might be useful for the detection of high-risk aortic plaques in patients with cryptogenic stroke.

Original article Harloff A et al. (2007) Reliability of aortic MRI at 3Tesla in patients with acute cryptogenic stroke. J Neurol Neurosurg Psychiatry [doi:10.1136/ jnnp.2007.125211]

\section{Study demonstrates axonal transport of West Nile virus in vivo}

West Nile virus (WNV) is an important cause of epidemic viral encephalitis and flaccid limb paralysis, but the mechanism by which it enters the CNS has, until recently, remained a matter of some debate. Now, Samuel et al. have demonstrated that WNV undergoes axonal transport both in vitro and in vivo.

By use of neuronal cultures that comprised three compartments-a soma chamber, in which neurons were plated; a methylcellulose barrier; and a neurite chamber, where distal axons emerged and target cells were platedthe researchers demonstrated that WNV can spread in a retrograde and anterograde direction via axonal transport from neuron to neuron. Further experiments with Vero cells-initially as the target for, and then as the source of, WNV infection-demonstrated 